\title{
Stellenwert der Polymeraseketten-Reaktion (PCR) und anderer Amplifikationsverfahren in der Diagnostik der Tuberkulose
}

\author{
Gaby E. Pfyffer
}

\author{
Ranking of the Poymerase Chain Reaction (PCR) and of Other Amplification \\ Methods in the Diagnosis of Tuberculosis
}

\begin{abstract}
"The ability of the PCR procedure to amplify a target DNA segment in genomic DNA raises the possibility that its use may extend beyond that of prenatal diagnosis to other areas of molecular biology." Saiki et al. 1985.
\end{abstract}

\section{Zusammenfassung}

\section{Abstract}

Mit jährlich fast drei Millionen Toten und über 8 Millionen Neuerkrankungen (wovon 3,2\% mit multiresistenten Erregern) nimmt die Tuberkulose (TB) weltweit noch immer einen Spitzenplatz unter den Infektionskrankheiten ein. Dem klinischen Laboratorium kommt mit einem schnellen Nachweis der TB-Bakterien und der raschen Verfügbarkeit der Resistenzprüfung eine zentrale Rolle zu. Während vor rund 20 Jahren der Polymeraseketten-Reaktion (PCR) noch ein verhältnismäßig bescheidenes Potenzial in der medizinischen Diagnostik zugetraut wurde [1], so gehören heute diese Technik und weitere, auf der In-vitro-Vervielfältigung von Nukleinsäuren beruhende Verfahren („Amplifikationsverfahren“) zum selbstverständlichen Instrumentarium eines modernen Mykobakterienlabors. Diese schnellen Methoden erweisen sich als äußerst hilfreich für (i) den Direktnachweis von Mycobacterium tuberculosis-Komplex im klinischen Untersuchungsmaterial; (ii) die Identifizierung von Mykobakterien; (iii) die rasche Ermittlung von Resistenzen gegenüber Antituberkulotika; und (iv) die DNA-Typisierung von TB-Stämmen zwecks molekular-epidemiologischer Abklärungen.

Worldwide, tuberculosis (TB) remains one of the leading infectious diseases, accounting for nearly 3 million deaths and more than 8 million new cases annually. Among them are 3.2\% multidrug-resistant strains of Mycobacterium tuberculosis. In the control of the spread of TB clinical mycobacteriology laboratories play an essential role providing the clinicians with timely detection, isolation, identification, and drug susceptibility testing results for M. tuberculosis. In the past twenty years numerous molecular techniques have been introduced to comply with the obvious needs for a reliable and rapid diagnosis of the disease. Nucleic acid amplification-based assays, in particular, the polymerase chain reaction (PCR), allow (i) direct detection of M. tuberculosis complex in clinical specimens; (ii) identification of mycobacteria; (iii) detection of resistance of M. tuberculosis to antimicrobial agents; and (iv) DNA typing to answer questions such as reactivation of disease or exogenous reinfection, and to track transmission and internal laboratory contaminations.

Institutsangaben

Chefmikrobiologin und Leiterin des Instituts für Medizinische Mikrobiologie, Zentrum für LaborMedizin .

Kantonsspital Luzern, Luzern

Anmerkung

Der Inhalt dieses Manuskripts war Bestandteil eines Vortrags anlässlich des Jahreskongresses

der Deutschen Gesellschaft für Pneumologie am 28.03.2003 in München

Korrespondenzadresse

Prof. Dr. phil. II Gaby E. Pfyffer, FAMH · Institut für Medizinische

Mikrobiologie · Zentrum für LaborMedizin · Kantonsspital Luzern · 6000 Luzern 16 · Schweiz ·

E-mail: gaby.pfyffer@ksl.ch

Eingang: 8. Januar $2004 \cdot$ Angenommen: 30. Januar 2004

Bibliografie

Pneumologie 2004; 58: 160-164 @ Georg Thieme Verlag Stuttgart · New York .

ISSN 0934-8387 · DOI 10.1055/s-2004-818379 
Amplifikationsverfahren für den Direktnachweis einer Tuberkulose aus klinischem Untersuchungsmaterial

Der molekularbiologische Direktnachweis von TB-Bakterien in klinischen Materialien stellt einen der signifikantesten Fortschritte in der Mykobakteriologie dar, ermöglicht er doch theoretisch eine Diagnosestellung innerhalb des gleichen Tages, an welchem die Probe im Labor eintrifft. Wie viele Labormethoden haben jedoch auch Amplifikationstechniken ihre Tücken und Unzulänglichkeiten, die sowohl Auftraggeber (Arzt) wie Anbieter (Laboratorium) kennen müssen. Richtig angewendet [2-5], sind sie eine willkommene Möglichkeit, TB-Patienten frühzeitig zu erfassen und entsprechende Maßnahmen einzuleiten.

Gegenwärtig bieten sich mehrere Amplifikationsverfahren für den Schnellnachweis einer TB an. Die PCR vervielfältigt (amplifiziert) in vitro mykobakterielle DNA, während das TMA-Verfahren (transcription-mediated amplification) mykobakterielle RNA amplifiziert. Für die PCR existieren eine Reihe individueller Laborprotokolle („inhouse PCR“), sie ist aber auch, wie die TMA, im Kit-Format kommerziell erhältlich (PCR: Amplicor ${ }^{\circledR}$ [Roche Diagnostic Systems, Indianapolis, In.]; TMA: MTD [Gen-Probe, San Diego, Ca.]). Eine weitere Amplifikationstechnik zur Diagnose einer TB ist die SDA (strand displacement amplification [Becton Dickinson Microbiology Systems, Sparks, Md.]). All diesen Verfahren ist gemeinsam, dass sie Mycobacterium tuberculosis-Komplex (d.h. M. tuberculosis, M. bovis ssp. bovis, M. bovis ssp. caprae, M. bovis BCG, M. africanum, M. microti, M. canettii) nachweisen, innerhalb des Komplexes aber nicht zu diskriminieren vermögen.

Mittlerweise sind hunderte von Studien publiziert, in welchen vor allem respiratorische Proben analysiert wurden. Sie zeigen generell, dass sowohl Sensitivität als auch Spezifität dieser Techniken für mikroskopisch-positive Materialien, d.h., für klinische Proben, welche $\geq 10^{4}$ TB-Bakterien $/ \mathrm{ml}$ enthalten, sehr hoch sind ( $\geq 96 \%$ resp. $\geq 99 \%$ ). Dies bedeutet, dass Fehldiagnosen eher eine Rarität sind. Anders verhält es sich mit mikroskopisch-negativen Materialien. Unabhängig vom gewählten Amplifikationsverfahren fällt hier vor allem die niedrigere Sensitivität (gemäß Literatur zwischen ca. 48-80\%) ins Gewicht, welche somit nur wenig besser als jene der Mikroskopie ist, während die Spezifität je nach Studie zwischen 95 und $98 \%$ liegt. Insbesondere in der paucibacillären Situation ist die Analyse von bis zu drei Proben angezeigt, da dadurch die Sensitivität gesteigert wird [6]. Gleiches trifft auch für extrapulmonale Untersuchungsmaterialien $\mathrm{zu}$, welche mehrheitlich mikroskopisch negativ sind. Bei den in den vielen Studien gemachten Angaben zu Sensitivität und Spezifität ist zu beachten, dass die überwiegende Mehrheit der Ergebnisse in Ländern mit niedriger TB-Prävalenz generiert worden sind, was den positiven resp. negativen prädiktiven Wert für solche Testverfahren wesentlich mitbeeinflusst.

Für den Kliniker wie auch für das Laborpersonal gilt es, die mittels Schnelltests erhaltenen Resultate richtig zu interpretieren und sich mit den neu daraus ergebenden Fragestellungen auseinanderzusetzen. Schwierigkeiten treten insbesondere dann auf, wenn die Ergebnisse eines molekularen Nachweisverfahrens von jenen der konventionellen Diagnostik (d.h. Kultur) resp. von der klinischen Verdachtsdiagnose divergieren. Negative Am- plifikationsresultate bei positiver Kultur können durch mögliche Inhibitoren im klinischen Untersuchungsmaterial oder aber durch eine inadäquate Verteilung der Bakterien beim Pipettieren verursacht werden. Ferner ist die Empfindlichkeit auch abhängig von der Art des abgenommenen Materials, des Probenvolumens und der Extraktionsmethode. Das Umgekehrte - Amplifikation positiv, Kultur negativ - stellt die Qualität des verwendeten Kulturverfahrens infrage oder aber lassen Befürchtungen einer möglichen laborinternen Kontamination aufkommen. Die Erfahrung zeigt jedoch, dass es sich nicht immer um ein „falsch-positives“ Ergebnis handeln muss! Dies gilt insbesondere, wenn das Untersuchungsmaterial von einem TB-Patienten unter Therapie stammt oder von einem Patienten, dessen klinische Symptomatik durchaus mit einer TB vereinbar ist, die Kultur aber aus irgend einem Grund negativ geblieben ist. Schwieriger wird die Interpretation divergenter Laborresultate bei symptomatischen Patienten, z.B. dann, wenn in der Anamnese eine vor langer Zeit durchgemachte TB vorliegt, resp. wenn ein enger Kontakt zu Patienten mit einer aktiven TB stattgefunden hat.

Die Handhabung von Amplifikationsverfahren ist relativ einfach, sofern das Labor über entsprechende Expertise und vor allem über gut geschultes Personal verfügt. Dies bedingt auch eine regelmäßige Teilnahme an externen Qualitätskontrollen. Solche Testmaterialien versenden derzeit u.a. die INSTAND (Düsseldorf/D) und die Centers for Disease Control and Prevention (CDC; Atlanta, Ga./USA). Noordhoek u. Mitarb. [7] haben anhand einer Studie mit internationaler Beteiligung eindrücklich demonstriert, dass mit jedem Verfahren, egal ob TMA, inhouse PCR oder kommerziell erhältlicher PCR, sowohl einwandfreie wie auch schlechte Ergebnisse generiert werden können. Hauptprobleme waren dabei eher eine mangelnde Spezifität (falsch positive Ergebnisse) sowie fehlende Teilnahme der Labors an externen Ringversuchen.

Während der Einsatz dieser Techniken ursprünglich für ein generelles Screening im Labor vorgesehen war, der letztlich die aufwendige Kultur ersetzen sollte, lehrt die mittlerweile bald zehnjährige Erfahrung, dass nur eine gezielte, also selektive, Anwendung dieser molekularbiologischen Werkzeuge sinnvoll ist. Primär leisten sie bei der Ermittlung eines Erstbefundes wertvolle Hilfe. Nicht geeignet sind sie dagegen für eine Verlaufskontrolle von Patienten unter Therapie, wiesen doch nach mehr als einem Jahr noch immer $22 \%$ der klinisch geheilten TB-Patienten ein positives PCR- resp. SDA-Resultat auf [8]. Der Einsatz dieser Techniken beschränkt sich somit klar auf das Auffinden neuer TB-Patienten. Allgemein gute Erfahrungen macht man in jenen Situationen, wo (i) aufgrund der klinischen und röntgenologischen Befunde ein TB-Verdacht besteht, (ii) der Patient immunkompromittiert ist (immunsupprimierende Therapie [z. B. bei Transplantationspatienten], HIV-Infekt, etc.) oder (iii) in Hochrisikosituationen (Krankenhaus, Altersheim, Gefängnis, Drogenszene, etc.). Um Kliniker wie Labor eine Entscheidungshilfe zu bieten, haben nun auch die CDC Richtlinien für einen sinnvollen und effizienten Einsatz dieser Testverfahren erlassen [9]. 
Zum jetzigen Zeitpunkt herrscht klar der Konsens, dass die Diagnose einer TB nicht allein auf dem Ergebnis einer molekularen Schnellmethode basieren darf, da ein negatives PCR-Ergebnis eine Mykobakterieninfektion nicht ausschließt. Somit hat die Interpretation des Resultats nach wie vor im Vergleich mit den Ergebnissen der konventionellen Diagnostik, insbesondere aber mit den verfügbaren klinischen Daten (Anamnese, Status des Patienten, röntgenologische Untersuchung, Therapieerfolg, allenfalls Hauttest, etc.) zu erfolgen, was eine enge Zusammenarbeit zwischen Klinik und Labor voraussetzt.

\section{Identifizierung von Mykobakterien}

Mittels molekulargenetischer Techniken können heute mehr oder weniger alle der nahezu 100 Mykobakterienarten einwandfrei und schnell identifiziert werden [10]. Auch hier ist der Einsatz der PCR nicht mehr wegzudenken, da bekanntlich die gewünschten DNA-Fragmente zuerst in vitro amplifiziert werden müssen, bevor sie überhaupt einer weiteren Analyse zugänglich sind. Tatsächlich verfügt man heute mit der Möglichkeit, nach vorgängiger PCR der Zielsequenzen einzelne Gene zu sequenzieren (z. B. das 16S rRNA-Gen oder 23SrRNA-Gen), über Identifizierungswerkzeuge für Mykobakterien, die den konventionellen biochemischen Techniken weit überlegen sind. Immer ausgehend von einer vorgängigen PCR erlauben auch andere Methoden eine weitestgehend zuverlässige, molekulargenetische Identifizierung auf Speziesebene, so z. B. die Restriktionsenzym-Analyse des $65 \mathrm{kD}$ Hitzeschockprotein(hsp)-Gens. Mittels Line probe assay (INNO-LiPA Mycobacteria, Innogenetics, Zwignaarde/B; GenoType Mycobacterium, Hain Lifescience, Nehren/D) lassen sich mittlerweile neben M. tuberculosis-Komplex auch über ein Dutzend der häufigsten nicht-tuberkulösen Mykobakterien (NTM) bestimmen. Die meisten dieser molekularen Techniken sind vorab für die Identifizierung von NTM interessant, während sie innerhalb des M. tuberculosis-Komplexes keine weitere Differenzierung erlauben. Dafür mussten bis vor kurzem noch immer der Nitrat- und der Niacin-Test sowie allenfalls weitere phänotypische Untersuchungen veranlasst werden. In diesem Jahr ist ein weiterer Line probe assay (Genotype MTBC, Hain Lifescience) verfügbar geworden, der innert weniger Stunden - ebenfalls nach vorangehender PCR - zwischen M. tuberculosis/M. africanum II/M. canettii, M. bovis ssp. bovis, M. bovis ssp. caprae, M. bovis BCG, M. africanum I und M. microti zu differenzieren vermag.

\section{Resistenzprüfung}

Resistenz von Mitgliedern des M. tuberculosis-Komplexes gegenüber antimikrobiellen Agentien ist stets ein Resultat von Mutationen. Diese beschränken sich auf chromosomale DNA und involvieren nicht, wie in anderen Bakterien, auch mobile genetische Elemente (z.B. Plasmide). Die Aufschlüsselung der wichtigsten Resistenzmechanismen von M. tuberculosis gegenüber einer Vielzahl von Medikamenten gelang dank den heute verfügbaren molekulargenetischen Techniken [11]. Unter anderen ermöglichen die DNA-Sequenzierung, die „Single-stranded conformation polmorphism “ (SSCP)-Analyse, das Dideoxy-Fingerprinting, die Hybridisierung an fester Phase (line probe assay, DNA hybridized microarray) oder an flüssiger Phase (heteroduplex analysis), in TB-Bakterien die für eine Resistenz verantwortliche(n) Mutation(en) innert kürzester Zeit nachzuweisen. Vorderhand aber sind die meisten dieser Verfahren dem Forschungslabor vorenthalten. Einzige Ausnahme bildet der kommerziell erhältliche Rifampicin Line Probe Assay (INNO LiPA Rif. TB, Innogenetics) [12]. Mit dieser Methode kann einfach und innerhalb von wenigen Stunden eine Resistenz gegenüber Rifampicin festgestellt werden, und zwar mittels einer OligonukleotidSonde, welche M. tuberculosis-Komplex nachweist, und durch neun weitere Sonden, welche $98 \%$ der für die Rifampicin-Resistenz verantwortlichen Punktmutationen auf dem rpoB-Gen erfassen. Der Test funktioniert tadellos, sofern er ab Kultur oder klinischem Material mit mikroskopisch reichlich säurefesten Stäbchen durchgeführt wird.

Die Einführung einer generellen Resistenzbestimmung mittels molekularer Techniken scheint angesichts der Zeitersparnis eine vielversprechende und erstrebenswerte Option, welche gegenüber den konventionellen Resistenzprüfungsverfahren überlegen ist. Mit Ausnahme des Nachweises einer Rifampicin-Resistenz (s. oben) sind aber gegenwärtig die molekularen Verfahren zur Resistenzbestimmung im Routinelabor nicht realistisch, dies vor allem aus zwei Gründen: (1) Nicht wie bei der Rifampicinresistenz, wo sich die Mutationen auf eine verhältnismäßig kurze Sequenz in einem einzigen Gen beschränken, ist die Resistenz gegenüber den meisten anderen Antituberkulotika durch Mutationen in verschiedenen Loci innerhalb eines Gens oder sogar in mehreren verschiedenen Genen bedingt, wodurch multiple Assays vonnöten sind. (2) Bis dato sind bei weitem nicht alle Resistenzmechanismen gegen einzelne TB-Medikamente (und dazugehörige Gene) entschlüsselt. Aus diesem Grund kann ein Fehlen einer Mutation in einem bestimmten Gen nicht mit einer „Empfindlichkeit“ des TB-Erregers gleichgesetzt werden, dies mit der Konsequenz, dass die mit molekularen Methoden generierten Resultate bis heute stets mit phänotypischen Resistenzprüfungen (zumeist via Kultur) bestätigt werden müssen.

\section{Molekulare Epidemiologie der Tuberkulose}

Will man die Frage einer Reaktivierung einer TB oder einer exogenen Reinfektion bei einem ehemaligen TB-Patienten abklären, muss der Erreger näher typisiert werden. Gleiches gilt für die Aufdeckung möglicher Übertragungswege dieser Krankheit. Dies ist vor allem sinnvoll bei „Mikroepidemien“ in Spitälern, Gefängnissen, Alters- und Obdachlosenheimen oder in anderen Risikosituationen. Eine Typisierung von M. tuberculosis ist ferner indiziert bei vermuteter Kreuzkontamination, z.B. nach einer Bronchoskopie oder im Diagnostiklabor.

Lange boten sich für M. tuberculosis nur die Phagen- und Serotypisierung an, zwei wenig effiziente und mit weiteren Schwierigkeiten verbundene Techniken. Dank der PCR kann man heute zuverlässig genetische Fingerabdrücke (DNA-fingerprints) herstellen. Die heutige Standardmethode basiert auf der Tatsache, dass im Genom von M. tuberculosis zwar keine Plasmide vorkommen, wohl aber repetitive Elemente, sog. Insertionssequenzen (IS), welche molekularbiologisch gut charakterisiert sind und sich als zuverlässige Marker bewährt haben [13]. Am bekanntesten 
für M. tuberculosis-Komplex ist die IS6110, von welcher M. tuberculosis durchschnittlich 5-18 Kopien, M. bovis dagegen nur einige wenige Kopien enthält. Schneidet man solche Sequenzen mit entsprechenden Restriktionsenzymen („genetischen Scheren“), so zeigt jeder Stamm eine für ihn charakteristische Anzahl IS-Kopien, welche ihrerseits von genau definierter Größe (Fragmentlänge) sind. Mit diesen beiden Diskriminanten, Kopiezahl und Fragmentlänge (Restriktionsfragmentlängen-Polymorphismus, RFLP), sind einzelne Stämme von M. tuberculosis leicht voneinander unterscheidbar. Dabei fasst man Stämme mit identischem IS6110-Bandenmuster in sog. „Cluster“ zusammen. In der Regel liefert eine RFLP-Analyse zuverlässige Resultate, sofern die Anzahl der IS6110-Kopien >5 ist. Unterschreitet die Zahl der Fragmente aber diese Größe, wie dies etwa von asiatischen M. tuberculosis-Stämmen, M. bovis und anderen Raritäten des M. tuberculosis-Komplexes (M. canettii) der Fall ist, empfiehlt sich die Verwendung zusätzlicher genetischer Marker (z. B. polymorphic guanine-cytosine-rich repetitive sequence [PGRS; 14]) oder anderer DNA-Typisierungsmethoden wie z.B. das Spoligotyping [15].

Die heute etablierten molekularen Verfahren eignen sich in erster Linie für die Beantwortung der eingangs erwähnten Fragestellungen, dienen also nicht zur Diagnosestellung einer TB aus klinischem Primärmaterial. Mit der Standardisierung der IS6110-RFLP-Technik und der Entwicklung spezieller SoftwarePakete können außerdem umfangreiche Datenbanken im Labor generiert werden. Dies erlaubt weltweit das Studium großer Patientenkohorten, aber auch das Auffinden von TB-Stämmen, welche durch besondere Virulenz auffallen (z.B. W-Stamm [16]). Die Literatur zu diesem Thema ist sehr umfangreich geworden. So weiß man von TB-Ausbrüchen in Kliniken, oft von multiresistenten M. tuberculosis-Erregern verursacht, bei welchen vor allem HIV-positive Patienten, aber auch Mitarbeiter solcher Institutionen involviert waren [17]. Andere Studien konzentrieren sich auf das Problem der Ansteckung in Gefängnissen [18] oder in bestimmten Risikogruppen (Drogenabhängige, Obdachlose, Alkoholiker $[19,20])$. Diese Arbeiten zeigen, dass dem DNAFingerprinting vom epidemiologischen Standpunkt her eine wichtige Rolle zukommt, trägt es doch zur Senkung der TB-Inzidenz, und, damit verbunden, zur Unterbrechung von Infektketten bei.

Schließlich sei erwähnt, dass sich diese molekular-epidemiologischen Techniken auch zu Identifizierung von zwei selten gesehenen Mitgliedern des M. tuberculosis-Komplexes eignen. Ursprünglich in verschiedenen Nagetieren und anderen Warmblütern nachgewiesen, ist M. microti vermehrt auf das Interesse des Klinikers gestoßen, nachdem 1998 erstmals vier Patienten mit einer durch diesen Keim bedingten, klinisch manifesten TB beschrieben worden waren [21]. Wohl kann die speziell gebogene Stäbchenmorphologie in der säurefesten Färbung auf M. microti hinweisen, ebenso die Tatsache, dass der Organismus in Kultur praktisch nicht anzüchtbar ist. Somit ist es in den meisten Fällen nicht möglich, eine Speziesdiagnose mit konventionellen biochemischen Tests vorzunehmen. Stattdessen kann M. microti mit dem Spoligotyping aufgrund seines charakteristischen Musters identifiziert werden. Auch für eine Speziesdiagnose von M. canettii, dem jüngsten Mitglied des M. tuberculosis-Komplexes [22 - 24], reichen konventionelle Identifizierungskriterien nicht.
Der Organismus kann, ebenfalls wie M. microti, nur mit gezielter RFLP und Spoligotyping erkannt werden.

\section{Literatur}

${ }^{1}$ Saiki RK, Scharf F, Faloona F et al. Enzymatic amplification of beta-globin genomic sequences and restriction site analysis for diagnosis of sickle cell anemia. Science 1985; 230: 1350-1354

2 Böttger EC, Feldmann K, Pfyffer GE et al. Molekularbiologische Methoden im mykobakteriologischen Laboratorium - im Jahre 1995 ein Fortschritt, ein Problem oder beides? Pneumologie 1995; 49: 664-666

3 Pfyffer GE. Nucleic acid amplification for mycobacterial diagnosis. J Infect 1999; 39: 21-26

${ }^{4}$ Della-Latta P, Whittier S. Comprehensive evaluation of performance, laboratory application, and clinical usefulness of two direct amplification technologies for the detection of Mycobacterium tuberculosis complex. Am J Clin Pathol 1998; 10: 301 - 310

${ }^{5}$ Forbes B, Pfyffer GE. Molecular diagnosis of mycobacterial infections. In: Jacobs WR, Cole S, Eisenach KD, Gicquel B, McMurray D (Hrsg). Tuberculosis. 2. Aufl. Washington, DC: ASM Press, 2004 (im Druck)

${ }^{6}$ Pfyffer GE, Mathews G, Brundney K et al. Performance of the Amplified Mycobacterium Tuberculosis Direct Test (MTD) on a by patient basis and a by specimen basis assessing the need for evaluating multiple specimens. (Eingereicht.)

${ }^{7}$ Noordhoek GT, Embden JDA van, Kolk AHJ. Reliability of nucleic acid amplification for detection of Mycobacterium tuberculosis: an international collaborative quality control study among 30 laboratories. J Clin Microbiol 1996; 34: 2522-2525

${ }^{8}$ Hellyer TJ, Flechter TW, Bates JH et al. Strand displacement amplification and the polymerase chain reaction for monitoring response to treatment in patients with pulmonary tuberculosis. J Infect Dis 1996; 173: $934-941$

${ }^{9}$ Centers for Disease Control and Prevention. Update: Nucleic acid amplification tests for tuberculosis. Morbid Mortal Wkl Rep 2000; 49: $593-594$

${ }^{10}$ Vincent V, Brown-Elliott BA, Jost KC et al. Mycobacterium: Phenotypic and genotypic identification. In: Murray PR, Baron EJ, Jorgensen $\mathrm{JH}$, Pfaller MA, Yolken RH (Hrsg). Manual of Clinical Microbiology, 8. Aufl. Washington, DC: ASM Press, 2003: 560-584

${ }^{11}$ Zhang Y, Telenti A. Genetics of drug resistance in Mycobacterium tuberculosis. In: Hatfull G, Jacobs WR (Hrsg). Molecular Genetics of Mycobacteria. Washington DC: ASM Press, 2000: 235-254

12 Rossau R, Traore H, de Beenhouwer $\mathrm{H}$ et al. Evaluation of the INNOLiPA Rif. TB Assay for the simultaneous detection of Mycobacterium tuberculosis and its resistance to rifampin. Antimicrob Agents Chemother 1997; 41: 2093-2098

${ }^{13}$ Embden JDA van, Cave MD, Crawford JT et al. Strain identification of Mycobacterium tuberculosis by DNA fingerprinting: recommendations for standardized methodology. J Clin Microbiol 1993; 31: 406-409

${ }^{14}$ Poulet S, Cole ST. Characterization of the highly abundant polymorphic GC-rich-repetitive sequence (PGRS) present in Mycobacterium tuberculosis. Arch Microbiol 1995; 163: 79-86

${ }^{15}$ Kamerbeek J, Schouls L, Kolk A et al. Simultaneous detection and strain differentiation of Mycobacterium tuberculosis for diagnosis and epidemiology. J Clin Microbiol 1997; 35: 907-914

${ }^{16}$ Agerton TB, Valway SE, Blinkhorn RJ et al. Spread of strain W, a highly drug-resistant strain of Mycobacterium tuberculosis, across the United States. Clin Infect Dis 1999; 29: 85-92

${ }^{17}$ Beck-Sagué C, Dooley SW, Hutton MD et al. Hospital outbreak of multidrug-resistant Mycobacterium tuberculosis infections. JAMA 1992; 268: $1280-1286$

${ }^{18}$ Pfyffer GE, Strässle A, Gorkum T van et al. Multi-drug resistant tuberculosis in prison inmates, Azerbaijan. Emerg Infect Dis 2001; 7: $855-861$

19 Barnes PF, El Hajj H, Preston-Martin S et al. Transmission of tuberculosis among the urban homeless. JAMA 1996; 275: $305-307$

${ }^{20}$ Genewein A, Telenti A, Bernasconi C et al. Molecular approach to identifying route of transmission of tuberculosis in the community. Lancet 1993; 342 : $841-844$ 
${ }^{21}$ Soolingen D van, Zanden AGM van der, de Haas PEW et al. Diagnosis of Mycobacterium microti infections among humans by using novel genetic markers. J Clin Microbiol 1998; 36: 1840-1845

${ }^{22}$ Soolingen D van, Hoogenboezem T, de Haas PEW et al. A novel pathogenic taxon of the Mycobacterium tuberculosis complex, Canettii: characterization of an exceptional isolate from Africa. Int J Syst Bacteriol 1997; 47: $1236-1245$
${ }^{23}$ Pfyffer GE, Auckenthaler R, Embden JDA van et al. Mycobacterium canettii, the smooth variant of M. tuberculosis, isolated from a Swiss patient exposed in Africa. Emerg Infect Dis 1998; 4: 631-634

${ }^{24}$ Miltgen J, Morillon M, Koeck JL et al. Two cases of pulmonary tuberculosis caused by Mycobacterium tuberculosis subsp. canettii. Emerg Infect Dis 2002; 8: 1350-1352

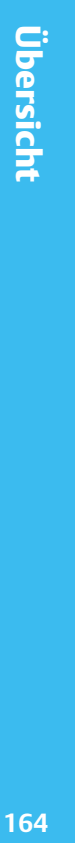

\section{Sleep Medicine Pearls}

R. B. Berry

Hanley \& Belfus, Philadelphia 2003, 388 S. \$39,95,

ISBN 1-56053-490-7

Sucht man ein praxisnahes Lehrbuch der Schlafmedizin, wird man in der deutschsprachigen Fachliteratur kaum fündig. Der Bedarf für eine umfassende und dabei gut lesbare Einführung in die Schlafmedizin ist zweifellos vorhanden, bedenkt man, welche Verbreitung Schlaflabore in Deutschland in den letzten Jahren erlebt haben. Nun ist es gewiss nicht leicht, das etwas trockene und abstrakte Gebiet der Schlafmedizin didaktisch gut aufzubereiten. Richard Berry ist dies in seinem Buch „Sleep Medicine Pearls" dennoch ausgezeichnet gelungen. Das Buch ist daher auch für den deutschen Leser zu empfehlen. Berry verwendet einen selbst für den im Englischen weniger versierten Leser gut verständlichen Stil.

In 22 kurzen Kapiteln wird eine Einführung in die Grundbegriffe der Schlafmedizin und die einschlägigen Untersuchungsverfahren sowie ein Überblick über die schlafmedizinisch relevanten Krankheitsbilder gegeben. Jedem Kapitel folgen mehrere Falldarstellungen aus der schlafmedizinischen Praxis, die den vorangegangenen Stoff illustrieren und vertiefen. Dabei wird der Leser zum aktiven Mitdenken angehalten, indem für den jeweils vorgestellten Patienten Diagnose oder Behandlungsvorschläge anzugeben sind. In der ausführlichen Diskussion, die sich jedem Fallbericht anschließt, wird das Krankheitsbild praxisrelevant erläutert, das jeweilige Einführungskapitel zum Teil wiederholt und um detaillierte Ausführungen ergänzt. Am Ende jeder Diskussion findet sich eine Zusammenfassung in Form einiger weniger Merksätze. Die einzelnen Falldarstellungen sind so gehalten, dass sie auch für sich verständlich sind. Will man z.B. für eine Prüfung den Stoff in Kürze rekapitulieren, arbeitet man lediglich die Merksätze am Ende jedes Kapitels durch.

Das Buch führt zunächst in die Klassifikation der Schlafstadien und der respiratorischen Ereignisse ein, widmet sich dann ausführlich der Technik der Polysomnografie und des Multiplen
Schlaf-Latenz-Tests. Anschließend werden das Obstruktive Schlafapnoesyndrom, seine Behandlungsmöglichkeiten und die Probleme, die bei den verschiedenen therapeutischen Optionen, insbesondere bei der CPAP-Therapie auftreten können, eingehend dargestellt. Weitere Kapitel behandeln das Zentrale Schlafapnoesyndrom, das Restless-Legs-Syndrom, das Periodic-legmovement-Syndrom, die Narkolepsie, ferner Parasomnien, nächtliche Epilepsien und die Abklärung der Insomnie. Eindrucksvolle und praxisnahe Schilderungen von insgesamt 112 Patienten verdeutlichen das jeweilige Thema. In den Falldiskussionen wird ausführlich auf Differentialdiagnostik und therapeutische Optionen der genannten Krankheitsbilder eingegangen.

Berry spricht auch neuere Behandlungsverfahren, wie die AutoCPAP-Therapie beim Obstruktiven Schlafapnoesyndrom und die adaptive Servo-Ventilation bei der Cheyne-Stokes-Atmung. Einigen Kapiteln merkt man die Herkunft des Autors aus den USA an. So wird z.B. der Uvulopalatopharyngoplastie mehr Bedeutung zugemessen als dies in Deutschland üblich ist. Auch dürften sogenannte „Split-night“-Untersuchungen in den USA gängiger sein als hierzulande. Der von Berry beschriebene „Maintenance of Wakefulness"-Test wird sicher bei uns kaum angewendet. In diesem Zusammenhang ist kritisch anzumerken, dass das forensisch wichtige Thema der Fahrtüchtigkeit beim Schlafapnoesyndrom etwas kurz geraten ist. Mit Überraschung nimmt man schließlich zur Kenntnis, dass die Somnoplastie als Therapieverfahren beim Obstruktiven Schlafapnoesyndrom keine Erwähnung findet.

Insgesamt stellt der Autor jedoch die theoretischen Grundlagen der Schlafmedizin umfassend und didaktisch gut dar. Die Schlafstadienanalyse und die polysomnografische Messung werden so ausführlich besprochen, dass der Leser ohne Zweifel nach der Lektüre selbständig Schlafstadien und Polysomnogramme beurteilen kann. Natürlich hilft es zum Verständnis des Textes, wenn man bereits erste Erfahrungen im Schlaflabor gesammelt hat. Schließlich sind die detaillierten und praxisnahen Fallbesprechungen als großes Plus des Buches hervorzuheben.

Christoph Stolpe, Essen 